TRANSACTIONS OF THE

AMERICAN MATHEMATICAL SOCIETY

Volume 365, Number 1, January 2013, Pages 123-141

S 0002-9947(2012)05505-X

Article electronically published on June 27, 2012

\title{
SHARP ILL-POSEDNESS AND WELL-POSEDNESS RESULTS FOR THE KdV-BURGERS EQUATION: THE PERIODIC CASE
}

\author{
LUC MOLINET AND STÉPHANE VENTO
}

\begin{abstract}
We prove that the KdV-Burgers equation is globally well-posed in $H^{-1}(\mathbb{T})$ with a solution-map that is analytic from $H^{-1}(\mathbb{T})$ to $C\left([0, T] ; H^{-1}(\mathbb{T})\right)$, whereas it is ill-posed in $H^{s}(\mathbb{T})$, as soon as $s<-1$, in the sense that the flowmap $u_{0} \mapsto u(t)$ cannot be continuous from $H^{s}(\mathbb{T})$ to even $\mathcal{D}^{\prime}(\mathbb{T})$ at any fixed $t>0$ small enough. In view of the result of Kappeler and Topalov for KdV it thus appears that even if the dissipation part of the KdV-Burgers equation allows us to lower the $C^{\infty}$ critical index with respect to the $\mathrm{KdV}$ equation, it does not permit us to improve the $C^{0}$ critical index.
\end{abstract}

\section{INTRODUCTION AND MAIN RESULTS}

The aim of this paper is to establish positive and negative optimal results on the Cauchy problem in Sobolev spaces for the Korteweg-de Vries-Burgers (KdV-B) equation posed on the one dimensional torus $\mathbb{T}=\mathbb{R} / 2 \pi \mathbb{Z}$ :

$$
u_{t}+u_{x x x}-u_{x x}+u u_{x}=0
$$

where $u=u(t, x)$ is a real-valued function.

This equation has been derived by Ott and Sudan [17] as an asymptotic model for the propagation of weakly nonlinear dispersive long waves in some physical contexts when dissipative effects occur.

In order to make our result more transparent, let us first introduce different notions of well-posedness (and consequently ill-posedness) related to the smoothness of the flow-map (see in the same spirit [13, [10]). Throughout this paper we shall say that a Cauchy problem is (locally) $C^{0}$-well-posed in some normed function space $X$ if, for any initial data $u_{0} \in X$, there exist a radius $R>0$, a time $T>0$ and a unique solution $u$, belonging to some space-time function space continuously embedded in $C([0, T] ; X)$, such that for any $t \in[0, T]$ the map $u_{0} \mapsto u(t)$ is continuous from the ball of $X$ centered at $u_{0}$ with radius $R$ into $X$. If the map $u_{0} \mapsto u(t)$ is of class $C^{k}$, $k \in \mathbb{N} \cup\{\infty\}$ (resp. analytic), we will say that the Cauchy is $C^{k}$-well-posed (resp. analytically well-posed). Finally, a Cauchy problem will be said to be $C^{k}$-ill-posed, $k \in \mathbb{N} \cup\{\infty\}$, if it is not $C^{k}$-well-posed.

In [16, Molinet and Ribaud proved that this equation is analytically well-posed in $H^{s}(\mathbb{T})$ as soon as $s>-1$. They also established that the index -1 is critical for the $C^{2}$-well-posedness. The surprising part of this result was that the $C^{\infty}$ critical index $s_{c}^{\infty}(K d V B)=-1$ was lower than the one of the $\mathrm{KdV}$ equation

$$
u_{t}+u_{x x x}+u u_{x}=0
$$

Received by the editors March 28, 2010 and, in revised form, November 15, 2010.

2010 Mathematics Subject Classification. Primary 35E15; Secondary 35M11, 35Q53, 35Q60.

(C)2012 American Mathematical Society Reverts to public domain 28 years from publication 
for which $s_{c}^{\infty}(K d V)=-1 / 2$ (cf. [14, 7]) and also lower than the $C^{\infty}$ index $s_{c}^{\infty}(d B)=s_{c}^{0}(d B)=-1 / 2$ (cf. [1], [9]) of the dissipative Burgers equation

$$
u_{t}-u_{x x}+u u_{x}=0 \text {. }
$$

On the other hand, using the integrability theory, it was recently proved in 13 that the flow-map of the KdV equation can be uniquely continuously extended in $H^{-1}(\mathbb{T})$. Therefore, on the torus, $\mathrm{KdV}$ is $C^{0}$-well-posed in $H^{-1}$ if one takes as a uniqueness class the class of a strong limit in $C\left([0, T] ; H^{-1}(\mathbb{T})\right)$ of smooth solutions.

In [15] the authors completed the result of [16] in the real line case by proving that the KdV-Burgers equation is analytically well-posed in $H^{-1}(\mathbb{R})$ and $C^{0}$-illposed in $H^{s}(\mathbb{R})$ for $s<-1$ in the sense that the flow-map defined on $H^{-1}(\mathbb{R})$ is not continuous for the topology inducted by $H^{s}, s<-1$, with values even in $\mathcal{D}^{\prime}(\mathbb{R})$. To reach the critical Sobolev space $H^{-1}(\mathbb{R})$ they adapted the refinement of Bourgain's spaces that appeared in [20] and [19] to the framework developed in [16. The proof of the main bilinear estimate used in a crucial way the Kato smoothing effect that does not hold on the torus. Our aim here is to give the new ingredients that enable one to overcome this lack of smoothing effects. The main idea is to weaken the space regularity of the Bourgain spaces in a suitable spacetime frequencies region. Note that our resolution space will still be embedded in $C\left([0, T] ; H^{-1}(\mathbb{T})\right)$ and that, to get the $L^{\infty}\left([0, T] ; H^{-1}(\mathbb{T})\right)$-estimate in this region, we use an idea that appeared in [4. Finally, once the well-posedness result is proved, the proof of the ill-posedness result follows exactly the same lines as in [15. It is due to a high to low frequency cascade phenomena that was first observed in 2] for a quadratic Schrödinger equation.

In view of the result of Kappeler and Topalov for KdV it thus appears that, at least on the torus, even if the dissipation part of the $\mathrm{KdV}$-Burgers equation ${ }^{1}$ allows one to lower the $C^{\infty}$ critical index with respect to the $\mathrm{KdV}$ equation, it does not permit one to improve the $C^{0}$ critical index.

Our results can be summarized as follows:

Theorem 1.1. The Cauchy problem associated to (1.1) is locally analytically wellposed in $H^{-1}(\mathbb{T})$. Moreover, at every point $u_{0} \in H^{-1}(\mathbb{T})$ there exist $T=T\left(u_{0}\right)>0$ and $R=R\left(u_{0}\right)>0$ such that the solution-map $u_{0} \mapsto u$ is analytic from the ball centered at $u_{0}$ with radius $R$ of $H^{-1}(\mathbb{T})$ into $C\left([0, T] ; H^{-1}(\mathbb{T})\right)$. Finally, the solution $u$ can be extended for all positive times and belongs to $C\left(\mathbb{R}_{+}^{*} ; H^{\infty}(\mathbb{T})\right)$.

Now that we have established analytic well-posedness, proceeding exactly as in [15. by taking as a sequence of initial data

$$
\phi_{N}(x)=N \cos (N x),
$$

we get the following ill-posedness result.

Theorem 1.2. The Cauchy problem associated to (1.1) is ill-posed in $H^{s}(\mathbb{T})$ for $s<-1$ in the following sense: there exists $T>0$ such that for any $0<t<T$, the flow-map $u_{0} \mapsto u(t)$ constructed in Theorem 1.1 is discontinuous at the origin from $H^{-1}(\mathbb{T})$ endowed with the topology inducted by $H^{s}(\mathbb{T})$ into $\mathcal{D}^{\prime}(\mathbb{T})$.

\footnotetext{
${ }^{1}$ It is important to notice that the dissipative term $-u_{x x}$ is of lower order than the dispersive one $u_{x x x}$.
} 
Remark 1.1. We could also be interested in a more general family of dissipative $\mathrm{KdV}$-type equations taking the form

$\left(g K d V B_{\alpha, k}\right) \quad u_{t}+u_{x x x}+\left(-\partial_{x x}\right)^{\alpha / 2} u+u^{k} u_{x}=0$

with $\alpha>0$ and $k \geq 1$. Such models were studied on the real line in 21] for $k=1$ and in 8 for $k=2$. Using the method introduced in [16], almost sharp results have been obtained on the $C^{\infty}$-well-posedness of the associated Cauchy problems. In the periodic case we are not aware of any existing results, but a rough analysis suggests for instance that $s_{c}^{\infty}\left(g K d V B_{2,2}\right)=-1 / 4$. Combined perhaps with some new ingredients, our present work could certainly be adapted to get the well-posedness in the critical space $H^{s_{c}^{\infty}}\left(g K d V B_{\alpha, k}\right)$ in both the real and periodic cases 2 However, it is unclear whether this space is also the critical one for the $C^{0}$-well-posedness.

\section{Resolution SPACE}

In this section we introduce a few notation and we define our functional framework.

For $A, B>0, A \lesssim B$ means that there exists $c>0$ such that $A \leq c B$. When $c$ is a small constant we use $A \ll B$. We write $A \sim B$ to denote the statement that $A \lesssim B \lesssim A$. For $u=u(t, x) \in \mathcal{S}^{\prime}\left(\mathbb{R} \times \mathbb{T}\right.$ ), we denote by $\widehat{u}$ (or $\mathcal{F}_{x} u$ ) its Fourier transform in space and by $\widetilde{u}$ (or $\mathcal{F} u$ ) the space-time Fourier transform of $u$. We consider the usual Lebesgue spaces $L^{p}, L_{x}^{p} L_{t}^{q}$ and abbreviate $L_{x}^{p} L_{t}^{p}$ as $L^{p}$. Let us define the Japanese bracket $\langle x\rangle=\left(1+|x|^{2}\right)^{1 / 2}$ so that the standard nonhomogeneous Sobolev spaces are endowed with the norm $\|f\|_{H^{s}}=\left\|\langle\nabla\rangle^{s} f\right\|_{L^{2}}$.

We use a Littlewood-Paley analysis. Let $\eta \in C_{0}^{\infty}(\mathbb{R})$ be such that $\eta \geq 0$, supp $\eta \subset$ $[-2,2], \eta \equiv 1$ on $[-1,1]$. We next define $\varphi(k)=\eta(k)-\eta(2 k)$. Any summations over capitalized variables such as $N, L$ are presumed to be dyadic, i.e., these variables range over numbers of the form $2^{\ell}, \ell \in \mathbb{N} \cup\{-1\}$. We set $\varphi_{\frac{1}{2}} \equiv \eta$, and for $N \geq 1$, $\varphi_{N}(k)=\varphi(k / N)$, and define the operator $P_{N}$ by $\mathcal{F}\left(P_{N} u\right)=\varphi_{N} \widehat{u}$. We introduce $\psi_{L}(\tau, k)=\varphi_{L}\left(\tau-k^{3}\right)$, and for any $u \in \mathcal{S}^{\prime}(\mathbb{R} \times \mathbb{T})$,

$$
\mathcal{F}_{x}\left(P_{N} u(t)\right)(k)=\varphi_{N}(k) \hat{u}(t, k), \quad \mathcal{F}\left(Q_{L} u\right)(\tau, k)=\psi_{L}(\tau, k) \tilde{u}(\tau, k) .
$$

Roughly speaking, the operator $P_{1 / 2}$ and $Q_{1 / 2}$ localize respectively in the ball $\{|k| \lesssim 1\}$ and $\left\{\left|\tau-k^{3}\right| \lesssim 1\right\}$, whereas for $N \geq 1$, the operator $P_{N}$ localizes in the annulus $\{|k| \sim N\}$ and $Q_{N}$ localizes in the region $\left\{\left|\tau-k^{3}\right| \sim N\right\}$.

Furthermore, we define more general projections $P_{\lesssim N}=\sum_{N_{1} \lesssim N} P_{N_{1}}, Q_{\gg L}=$ $\sum_{L_{1} \gg L} Q_{L_{1}}$, etc.

Let $e^{-t \partial_{x x x}}$ be the propagator associated to the Airy equation and define the two parameters linear operator $W$ by

$$
W\left(t, t^{\prime}\right) \phi=\sum_{k \in \mathbb{Z}} \exp \left(i t k^{3}-\left|t^{\prime}\right| k^{2}\right) \hat{\phi}(k) e^{i k x}, \quad t \in \mathbb{R} .
$$

The operator $W: t \mapsto W(t, t)$ is clearly an extension on $\mathbb{R}$ of the linear semi-group $S(\cdot)$ associated with (1.1) that is given by

$$
S(t) \phi=\sum_{k \in \mathbb{Z}} \exp \left(i t k^{3}-t k^{2}\right) \hat{\phi}(k) e^{i k x}, \quad t \in \mathbb{R}_{+} .
$$

\footnotetext{
${ }^{2}$ Note that the critical index $s_{c}^{\infty}$ may differ in the real and in the periodic cases.
} 
We will mainly work on the integral formulation of (1.1):

$$
u(t)=S(t) u_{0}-\frac{1}{2} \int_{0}^{t} S\left(t-t^{\prime}\right) \partial_{x} u^{2}\left(t^{\prime}\right) d t^{\prime}, \quad t \in \mathbb{R}_{+} .
$$

Actually, to prove the local existence result, we will follow the strategy of [15] and apply a fixed point argument to the following extension of (2.3):

$$
\begin{aligned}
u(t)=\eta(t)\left[W(t) u_{0}\right. & -\frac{1}{2} \chi_{\mathbb{R}_{+}}(t) \int_{0}^{t} W\left(t-t^{\prime}, t-t^{\prime}\right) \partial_{x} u^{2}\left(t^{\prime}\right) d t^{\prime} \\
& \left.-\frac{1}{2} \chi_{\mathbb{R}_{-}}(t) \int_{0}^{t} W\left(t-t^{\prime}, t+t^{\prime}\right) \partial_{x} u^{2}\left(t^{\prime}\right) d t^{\prime}\right] .
\end{aligned}
$$

It is clear that if $u$ solves (2.4), then $u$ is a solution of (2.3) on $[0, T], T<1$.

In [15], adapting some ideas of [20] and [19] to the framework developed in [16], the authors performed the iteration process in the sum space $X^{-1, \frac{1}{2}, 1}+Y^{-1, \frac{1}{2}}$, where

$$
\|u\|_{X^{s, b, 1}}=\left(\sum_{N}\left[\sum_{L}\langle N\rangle^{s}\left\langle L+N^{2}\right\rangle^{b}\left\|P_{N} Q_{L} u\right\|_{L_{x t}^{2}}\right]^{2}\right)^{1 / 2}
$$

and

$$
\|u\|_{Y^{s, b}}=\left(\sum_{N}\left[\langle N\rangle^{s}\left\|\mathcal{F}^{-1}\left[\left(i\left(\tau-k^{3}\right)+k^{2}+1\right)^{b+1 / 2} \varphi_{N} \widetilde{u}\right]\right\|_{L_{t}^{1} L_{x}^{2}}\right]^{2}\right)^{1 / 2},
$$

so that

$$
\|u\|_{Y^{-1, \frac{1}{2}}} \sim\left(\sum_{N}\left[\langle N\rangle^{-1}\left\|\left(\partial_{t}+\partial_{x x x}-\partial_{x x}+I\right) P_{N} u\right\|_{L_{t}^{1} L_{x}^{2}}\right]^{2}\right)^{1 / 2} .
$$

As explained in the introduction, due to the lack of the Kato smoothing effect on the torus, we will be able to control none of the two norms above in the region " $\sigma$-dominant". The idea is then to weaken the required $x$-regularity on the $X^{s, b}$ component of our resolution space in this region. For $\varepsilon>0$ small enough, we thus introduce the function space $X_{\varepsilon}^{s, b, q}$ endowed with the norm

$$
\begin{aligned}
\|u\|_{X_{\varepsilon}^{s, b, 1}}= & \left(\sum_{N}\left[\sum_{L \leq N^{3}}\langle N\rangle^{s}\left\langle L+N^{2}\right\rangle^{b}\left\|P_{N} Q_{L} u\right\|_{L_{x t}^{2}}\right]^{2}\right)^{1 / 2} \\
& +\left(\sum_{N}\left[\sum_{L>N^{3}}\langle N\rangle^{s-\varepsilon}\left\langle L+N^{2}\right\rangle^{b}\left\|P_{N} Q_{L} u\right\|_{L_{x t}^{2}}\right]^{2}\right)^{1 / 2} .
\end{aligned}
$$

However, $X_{\varepsilon}^{s, b, 1}$ is not embedded anymore in $L^{\infty}\left(\mathbb{R} ; H^{-1}(\mathbb{T})\right)$. For this reason we will take its intersection with the function space $\widetilde{L_{t}^{\infty} H^{-1}}$, that is, a dyadic version of $L^{\infty}\left(\mathbb{R} ; H^{-1}(\mathbb{T})\right)$ equipped with the norm

$$
\|u\|_{L_{t}^{\infty} H^{-1}}=\left(\sum_{N}\left[\langle N\rangle^{-1}\left\|P_{N} u\right\|_{L_{t}^{\infty} L_{x}^{2}}\right]^{2}\right)^{1 / 2} .
$$

Finally, we also need to define the space $Z^{s,-\frac{1}{2}}$ equipped with the norm

$$
\|u\|_{Z^{s,-\frac{1}{2}}}=\left(\sum_{N}\left[\langle N\rangle^{s}\left\|\varphi_{N}(k)\left\langle i\left(\tau-k^{3}\right)+k^{2}\right\rangle^{-1} \widetilde{u}\right\|_{L_{k}^{2} L_{\tau}^{1}}\right]^{2}\right)^{1 / 2} .
$$

We are now in a position to form our resolution space $\widetilde{\mathcal{S}_{\varepsilon}^{s}}=\left(X_{\varepsilon}^{s, \frac{1}{2}, 1} \cap \widetilde{L_{t}^{\infty} H^{-1}}\right)+Y^{s, \frac{1}{2}}$ and the "nonlinear space" $\mathcal{N}_{\varepsilon}^{s}=\left(X_{\varepsilon}^{s,-\frac{1}{2}, 1} \cap Z^{s,-\frac{1}{2}}\right)+Y^{s,-\frac{1}{2}}$, where the nonlinear 
term $\partial_{x} u^{2}$ will take place. Actually, we will estimate $\left\|\partial_{x} u^{2}\right\|_{\mathcal{N}_{\varepsilon}^{s}}$ in terms of $\|u\|_{\mathcal{S}_{\varepsilon}^{s}}$, where $\mathcal{S}_{\varepsilon}^{s}=X_{\varepsilon}^{s, \frac{1}{2}, 1}+Y^{s, \frac{1}{2}}$. Obviously $\|u\|_{\mathcal{S}_{\varepsilon}^{s}} \leq\|u\|_{\widetilde{\mathcal{S}}_{\varepsilon}^{s}}$, and the first of these norms has the advantage to only see the size of the modulus of the space-time Fourier transform of the function. This will be useful when dealing with the dual form of the main bilinear estimate.

Note that we endow these sum spaces with the usual norms:

$$
\|u\|_{X+Y}=\inf \left\{\left\|u_{1}\right\|_{X}+\left\|u_{2}\right\|_{Y}: u_{1} \in X, u_{2} \in Y, u=u_{1}+u_{2}\right\} .
$$

In the rest of this section, we study some basic properties of the function space $\mathcal{S}_{\varepsilon}^{-1}$.

Lemma 2.1. For any $\phi \in L^{2}$,

$$
\left(\sum_{L}\left[L^{1 / 2}\left\|Q_{L}\left(e^{-t \partial_{x x x}} \phi\right)\right\|_{L^{2}}\right]^{2}\right)^{1 / 2} \lesssim\|\phi\|_{L^{2}} .
$$

Proof. From Plancherel theorem, we have

$$
\left(\sum_{L}\left[L^{1 / 2}\left\|Q_{L}\left(e^{-t \partial_{x x x}} \phi\right)\right\|_{L^{2}}\right]^{2}\right)^{1 / 2} \sim\left\|\left|\tau-k^{3}\right|^{1 / 2} \mathcal{F}\left(e^{-t \partial_{x x x}} \phi\right)\right\|_{L^{2}}
$$

Moreover, if we set $\eta_{T}(t)=\eta(t / T)$ for $T>0$, then

$$
\mathcal{F}\left(\eta_{T}(t) e^{-t \partial_{x x x}} \phi\right)(\tau, k)=\widehat{\eta_{T}}\left(\tau-k^{3}\right) \widehat{\phi}(k) .
$$

Thus we obtain with the changes of variables $\tau-k^{3} \rightarrow \tau^{\prime}$ and $T \tau^{\prime} \rightarrow \sigma$ that

$$
\left\|\left|\tau-k^{3}\right|^{1 / 2} \mathcal{F}\left(\eta_{T}(t) e^{-t \partial_{x x x}} \phi\right)\right\|_{L^{2}} \lesssim\|\phi\|_{L^{2}}\left\|\left|\tau^{\prime}\right|^{1 / 2} T \widehat{\eta}\left(T \tau^{\prime}\right)\right\|_{L_{\tau^{\prime}}^{2}} \lesssim\|\phi\|_{L^{2}}
$$

Taking the limit $T \rightarrow \infty$, this completes the proof.

Lemma 2.2. (1) For any $\varepsilon \geq 0$ and all $u \in \widetilde{\mathcal{S}_{\varepsilon}^{-1}}$, we have

$$
\|u\|_{L_{t}^{\infty} H^{-1}(\mathbb{T})} \lesssim\|u\|_{\mathcal{S}_{\varepsilon}^{-1}} \quad \text { and } \quad\left(\sum_{N}\left\|P_{N} Q_{\leq N^{3}} u\right\|_{L_{t}^{\infty} H^{-1}(\mathbb{T})}^{2}\right)^{1 / 2} \lesssim\|u\|_{\mathcal{S}_{\varepsilon}^{-1}}
$$

(2) For any $0 \leq \varepsilon \leq 1 / 2$ and all $u \in \mathcal{S}_{\varepsilon}^{-1}$, we have

$$
\|u\|_{L_{x t}^{2}} \lesssim\|u\|_{\mathcal{S}_{\varepsilon}^{-1}}
$$

(3) For all $u \in Y^{0,1 / 2}$,

$$
\left(\sum_{L}\left[L^{1 / 2}\left\|Q_{L} u\right\|_{L^{2}}\right]^{2}\right)^{1 / 2} \lesssim\|u\|_{Y^{0, \frac{1}{2}}}
$$

Proof. (1) First it is fairly obvious that $\widetilde{L_{t}^{\infty} H_{x}^{-1}} \hookrightarrow L_{t}^{\infty} H_{x}^{-1}$ and that, according to the definition of $X_{\varepsilon}^{-1, \frac{1}{2}, 1}$,

$$
\left(\sum_{N}\left\|P_{N} Q_{\leq N^{3}} u\right\|_{L_{t}^{\infty} H^{-1}(\mathbb{T})}^{2}\right)^{1 / 2} \lesssim\|u\|_{X_{\varepsilon}^{-1, \frac{1}{2}, 1}} .
$$


Second, for any dyadic $N$,

$$
\begin{aligned}
\left\|P_{N} u\right\|_{L_{t}^{\infty} H^{-1}} & =\left\|\mathcal{F}_{t}^{-1}\left(\frac{\langle k\rangle^{-1}}{i\left(\tau-k^{3}\right)+k^{2}+1}\left(i\left(\tau-k^{3}\right)+k^{2}+1\right) \varphi_{N} \widetilde{u}\right)\right\|_{L_{t}^{\infty} L_{k}^{2}} \\
& \lesssim\left\|\mathcal{F}_{t}^{-1}\left(\frac{\langle k\rangle^{-1} \varphi_{N}(k)}{i\left(\tau-k^{3}\right)+k^{2}+1}\right)\right\|_{L_{t}^{\infty} L_{k}^{\infty}}\left\|P_{N} u\right\|_{Y^{0, \frac{1}{2}}} \\
& \lesssim\left\|\langle k\rangle^{-1} \varphi_{N}(k)\right\|_{L_{k}^{\infty}}\left\|e^{-t\langle N\rangle^{2}} \chi_{\mathbb{R}^{+}}(t)\right\|_{L_{t}^{\infty}}\left\|P_{N} u\right\|_{Y^{0, \frac{1}{2}}} \\
& \lesssim\langle N\rangle^{-1}\left\|P_{N} u\right\|_{Y^{0, \frac{1}{2}}} \lesssim\left\|P_{N} u\right\|_{Y^{-1, \frac{1}{2}}} .
\end{aligned}
$$

This completes the proof of (2.6) after square summing in $N$.

(2) In the same way, for any dyadic $N$,

$$
\begin{aligned}
\left\|P_{N} u\right\|_{L^{2}} & \lesssim \sum_{L \leq N^{3}}\left\|P_{N} Q_{L} u\right\|_{L^{2}}+\sum_{L>N^{3}}\left\|P_{N} Q_{L} u\right\|_{L^{2}} \\
& \lesssim \sum_{L \leq N^{3}}\left\|P_{N} Q_{L} u\right\|_{L^{2}}+\sum_{L>N^{3}} L^{1 / 2} N^{-3 / 2}\left\|P_{N} Q_{L} u\right\|_{L^{2}} \\
& \lesssim\left\|P_{N} u\right\|_{X_{1 / 2}^{-1, \frac{1}{2}, 1}} .
\end{aligned}
$$

On the other hand, applying Young and Hölder's inequalities, we get

$$
\begin{aligned}
\left\|P_{N} u\right\|_{L^{2}} & =\left\|\mathcal{F}_{t}^{-1}\left(\frac{1}{i\left(\tau-k^{3}\right)+k^{2}+1}\left(i\left(\tau-k^{3}\right)+k^{2}+1\right) \varphi_{N} \widetilde{u}\right)\right\|_{L_{t k}^{2}} \\
& \lesssim\left\|\mathcal{F}_{t}^{-1}\left(\frac{\varphi_{N}(k)}{i\left(\tau-k^{3}\right)+k^{2}+1}\right)\right\|_{L_{t}^{2} L_{k}^{\infty}}\left\|P_{N} u\right\|_{Y^{0, \frac{1}{2}}} \\
& \lesssim\left\|e^{-t\langle N\rangle^{2}} \chi_{\mathbb{R}^{+}}(t)\right\|_{L_{t}^{2}}\left\|P_{N} u\right\|_{Y^{0, \frac{1}{2}}} \\
& \lesssim\langle N\rangle^{-1}\left\|P_{N} u\right\|_{Y^{0, \frac{1}{2}}} \lesssim\left\|P_{N} u\right\|_{Y^{-1, \frac{1}{2}}} .
\end{aligned}
$$

This proves (2.7) after square summing in $N$.

(3) Setting $v=\left(\partial_{t}+\partial_{x x x}\right) u$, we see that $u$ can be rewritten as

$$
u(t)=e^{-t \partial_{x x x}} u(0)+\int_{0}^{t} e^{-\left(t-t^{\prime}\right) \partial_{x x x}} v\left(t^{\prime}\right) d t^{\prime} .
$$

By virtue of Lemma 2.1, we have

$$
\left(\sum_{L}\left[L^{1 / 2}\left\|Q_{L} e^{-t \partial_{x x x}} u(0)\right\|_{L^{2}}\right]^{2}\right)^{1 / 2} \lesssim\|u(0)\|_{L^{2}} \lesssim\|u\|_{L_{t}^{\infty} L_{x}^{2}}
$$

Moreover, we obtain as previously that

$$
\|u\|_{L_{t}^{\infty} L_{x}^{2}} \lesssim\left\|\mathcal{F}_{t}^{-1}\left(\frac{1}{i\left(\tau-k^{3}\right)+k^{2}+1}\right)\right\|_{L_{t k}^{\infty}}\|u\|_{Y^{0, \frac{1}{2}}} \lesssim\|u\|_{Y^{0, \frac{1}{2}}} .
$$

Now it remains to show that

$$
\left(\sum_{L}\left[L^{1 / 2}\left\|Q_{L} \int_{0}^{t} e^{-\left(t-t^{\prime}\right) \partial_{x x x}} v\left(t^{\prime}\right) d t^{\prime}\right\|_{L^{2}}\right]^{2}\right)^{1 / 2} \lesssim\|v\|_{L_{t}^{1} L_{x}^{2}}
$$


since the right-hand side is controlled by

$$
\begin{aligned}
\left\|P_{N} v\right\|_{L_{t}^{1} L_{x}^{2}} & \lesssim\left\|\left(I-\partial_{x x}\right) P_{N} u\right\|_{L_{t}^{1} L_{x}^{2}}+\left\|P_{N} u\right\|_{Y^{0, \frac{1}{2}}} \\
& \lesssim\left(\left\|\mathcal{F}_{t}^{-1}\left(\frac{\varphi_{N}(\xi)\left(\xi^{2}+1\right)}{i\left(\tau-\xi^{3}\right)+\xi^{2}+1}\right)\right\|_{L_{t}^{1} L_{\xi}^{\infty}}+1\right)\left\|P_{N} u\right\|_{Y^{0, \frac{1}{2}}} \\
& \lesssim\left\|P_{N} u\right\|_{Y^{0, \frac{1}{2}}} .
\end{aligned}
$$

In order to prove (2.10), we split the integral $\int_{0}^{t}=\int_{-\infty}^{t}-\int_{-\infty}^{0}$. By Lemma 2.1, the contribution with integrand on $(-\infty, 0)$ is bounded by

$$
\lesssim\left\|\int_{-\infty}^{0} e^{t^{\prime} \partial_{x x x}} v\left(t^{\prime}\right) d t^{\prime}\right\|_{L_{x}^{2}} \lesssim\|v\|_{L_{t}^{1} L_{x}^{2}}
$$

For the last term, we reduce by Minkowski to show that

$$
\left(\sum_{L}\left[L^{1 / 2}\left\|Q_{L}\left(\chi_{t>t^{\prime}} e^{-\left(t-t^{\prime}\right) \partial_{x x x}} v\left(t^{\prime}\right)\right)\right\|_{L_{t x}^{2}}\right]^{2}\right)^{1 / 2} \lesssim\left\|v\left(t^{\prime}\right)\right\|_{L_{x}^{2}}
$$

This can be proved by a time-restriction argument. Indeed, for any $T>0$, we have

$$
\begin{aligned}
& \left(\sum_{L}\left[L^{1 / 2}\left\|Q_{L}\left(\eta_{T}(t) \chi_{t>t^{\prime}} e^{-\left(t-t^{\prime}\right) \partial_{x x x}} v\left(t^{\prime}\right)\right)\right\|_{L^{2}}\right]^{2}\right)^{1 / 2} \\
& \quad \lesssim\left\||\tau|^{1 / 2} \widehat{v}\left(t^{\prime}\right) \mathcal{F}_{t}\left(\eta_{T}(t) \chi_{t>t^{\prime}}\right)(\tau)\right\|_{L^{2}} \\
& \quad \lesssim\left\|v\left(t^{\prime}\right)\right\|_{L^{2}}\left\||\tau|^{1 / 2} \mathcal{F}_{t}\left(\eta(t) \chi_{t T>t^{\prime}}\right)\right\|_{L^{2}} \\
& \quad \lesssim\left\|v\left(t^{\prime}\right)\right\|_{L^{2}} .
\end{aligned}
$$

We conclude by passing to the limit $T \rightarrow \infty$.

\section{Linear estimates}

It is straightforward to check that estimates on the linear operator $W(t)$ and on the extension of the Duhamel term proven in 15 on $\mathbb{R}$ still hold on $\mathbb{T}$. We thus will concentrate on the $X_{\varepsilon}^{s, \frac{1}{2}, 1} \cap \widetilde{L_{t}^{\infty} H^{-1}}$ component.

Proposition 3.1. (1) For any $\varepsilon \geq 0$ and all $\phi \in H^{-1}(\mathbb{T})$, we have

$$
\|\eta(t) W(t) \phi\|_{\widetilde{\mathcal{S}_{\varepsilon}^{-1}}} \lesssim\|\phi\|_{H^{-1}}
$$

(2) Let $\mathcal{L}: f \rightarrow \mathcal{L} f$ denote the linear operator

$$
\begin{aligned}
\mathcal{L} f(t, x)= & \eta(t)\left(\chi_{\mathbb{R}^{+}}(t) \int_{0}^{t} W\left(t-t^{\prime}, t-t^{\prime}\right) f\left(t^{\prime}\right) d t^{\prime}\right. \\
& \left.+\chi_{\mathbb{R}^{-}}(t) \int_{0}^{t} W\left(t-t^{\prime}, t+t^{\prime}\right) f\left(t^{\prime}\right) d t^{\prime}\right) .
\end{aligned}
$$

If $f \in \mathcal{N}_{\varepsilon}^{-1}$ with $\varepsilon \geq 0$, then

$$
\|\mathcal{L} f\|_{\widetilde{\mathcal{S}_{\varepsilon}^{-1}}} \lesssim\|f\|_{\mathcal{N}_{\varepsilon}^{-1}}
$$

Proof. The first assertion is a direct consequence of the corresponding estimate in $X^{s, \frac{1}{2}, 1}$, proven in [15] together with the continuous embedding $X^{s, \frac{1}{2}, 1} \hookrightarrow \widetilde{L_{t}^{\infty} H^{-1}} \cap$ $X_{\varepsilon}^{s, \frac{1}{2}, 1}$ for $\varepsilon \geq 0$. 
To prove the second assertion, it clearly suffices to show the following three inequalities:

$$
\begin{aligned}
\|\mathcal{L} f\|_{Y^{0, \frac{1}{2}}} & \lesssim\|f\|_{Y^{0,-\frac{1}{2}}}, \\
\|\mathcal{L} f\|_{X_{\varepsilon}^{0, \frac{1}{2}, 1}} & \lesssim\|f\|_{X_{\varepsilon}^{0,-\frac{1}{2}, 1}}+\|f\|_{Z^{0,-\frac{1}{2}}}, \\
\|\mathcal{L} f\|_{L_{t}^{\widetilde{\infty} H^{-1}}} & \lesssim\|f\|_{Z^{0,-\frac{1}{2}}} .
\end{aligned}
$$

Estimate (3.4) has been proved in [15. To prove (3.5) we first note that according to [15, it holds that

$$
\|\mathcal{L} f\|_{X^{s, \frac{1}{2}, 1}} \lesssim\|f\|_{X^{s,-\frac{1}{2}, 1}} .
$$

It is then not too hard to be convinced that (3.5) is a consequence of the following estimate:

$$
\begin{aligned}
& \left(\sum_{N \geq 4}\left[\sum_{L \leq N^{3}}\left\langle L+N^{2}\right\rangle^{1 / 2}\left\|P_{N} Q_{L}\left(\mathcal{L}\left(Q_{\geq 2 N^{3}} f\right)\right)\right\|_{L^{2}}\right]^{2}\right)^{1 / 2} \\
& \quad \lesssim\|f\|_{Z^{0,-\frac{1}{2}}}+\left(\sum_{N \geq 4}\left[\sum_{L=N^{3} / 2}^{N^{3}}\left\langle L+N^{2}\right\rangle^{-1 / 2}\left\|P_{N} Q_{L} f\right\|_{L^{2}}\right]^{2}\right)^{1 / 2} .
\end{aligned}
$$

To prove (3.6) and (3.8) we proceed as in 15. Using the $x$-Fourier expansion and setting $w(t)=U(-t) f(t)$, it is easy to derive that

$$
\mathcal{L} f(t, x)=U(t)\left[\eta(t) \sum_{k \in \mathbb{Z}} e^{i x k} \int_{\mathbb{R}} \frac{e^{i t \tau} e^{(t-|t|) k^{2}}-e^{-|t| k^{2}}}{i \tau+k^{2}} \tilde{w}(\tau, k) d \tau\right] .
$$

In particular, by Plancherel and Minkowski,

$$
\begin{aligned}
\|\mathcal{L} f\|_{{L_{t}^{\infty} L_{x}^{2}}} & =\|U(-t) \mathcal{L} f\|_{\widetilde{L_{t}^{\infty} L_{x}^{2}}} \\
& \leq\left(\sum_{N}\left\|\eta(t) \varphi_{N}(k) \int_{\mathbb{R}} \frac{e^{i t \tau} e^{(t-|t|) k^{2}}-e^{-|t| k^{2}}}{i \tau+k^{2}} \tilde{w}(\tau, k) d \tau\right\|_{L_{k}^{2} L_{t}^{\infty}}^{2}\right)^{1 / 2} .
\end{aligned}
$$

Now for $k \in \mathbb{Z}$ fixed and $v \in \mathcal{S}(\mathbb{R})$ we set

$$
K_{k}(v)(t)=\eta(t) \varphi_{N}(k) \int_{\mathbb{R}} \frac{e^{i t \tau} e^{(t-|t|) k^{2}}-e^{-|t| k^{2}}}{i \tau+k^{2}} v(\tau) d \tau .
$$

We thus are reduced to show that for any $k \in \mathbb{Z}$,

$$
\left\|K_{k}(v)\right\|_{L_{t}^{\infty}} \lesssim\left\|\frac{\varphi_{N}(k) v(\tau)}{\left\langle i \tau+k^{2}\right\rangle}\right\|_{L_{\tau}^{1}}
$$

and

$$
\begin{aligned}
\sum_{L \leq N^{3}}\left\langle L+N^{2}\right\rangle^{1 / 2}\left\|P_{L}\left(K_{k}\left(\Phi_{\geq 2 N^{3}} v\right)\right)\right\|_{L_{t}^{2}} \\
\quad \lesssim\left\|\frac{\varphi_{N}(k) v(\tau)}{\left\langle i \tau+k^{2}\right\rangle}\right\|_{L_{\tau}^{1}}+\sum_{L=N^{3} / 2}^{N^{3}} \varphi_{N}(k)\left\langle L+N^{2}\right\rangle^{-1 / 2}\left\|\varphi_{L} v\right\|_{L_{\tau}^{2}},
\end{aligned}
$$

where we set $\Phi_{\geq 2 N^{3}}:=\sum_{L \geq 2 N^{3}} \varphi_{L}$. To prove (3.9) it suffices to notice that

$$
\left\|K_{k}(v)\right\|_{L_{t}^{\infty}} \lesssim\|\eta\|_{L^{\infty} \varphi_{N}}(k) \int_{\mathbb{R}} \frac{|v(\tau)|}{\left|i \tau+k^{2}\right|} d \tau,
$$


which gives the result for $k \neq 0$. In the case $k=0$ we use a Taylor expansion to get

$$
\begin{aligned}
\left\|K_{0}(v)\right\|_{L_{t}^{\infty}} & \lesssim \varphi_{N}(k)\left[\|\eta\|_{L^{\infty}} \int_{|\tau| \geq 1} \frac{|v(\tau)|}{|\tau|} d \tau+\sum_{n \geq 1} \frac{1}{n !}\left\|t^{n} \eta\right\|_{L^{\infty}} \int_{|\tau| \leq 1} \frac{|\tau|^{n}|v(\tau)|}{|\tau|} d \tau\right] \\
& \lesssim \varphi_{N}(k) \int_{\mathbb{R}} \frac{|v(\tau)|}{\langle\tau\rangle} d \tau \sum_{n \geq 0} \frac{1}{n !}\left\|t^{n} \eta\right\|_{L^{\infty}}
\end{aligned}
$$

which is acceptable since $\left\|t^{n} \eta\right\|_{L^{\infty}} \lesssim 2^{n}$.

To get (3.10) we first rewrite $K_{k}\left(\Phi_{\geq 2 N^{3}} v\right)$ as

$$
\begin{aligned}
\eta(t) e^{(t-|t|) k^{2}} \varphi_{N}(k) \int_{\mathbb{R}} \frac{e^{i t \tau}}{i \tau+k^{2}} \Phi_{\geq 2 N^{3}}(\tau) v(\tau) d \tau & \\
& -\eta(t) \varphi_{N}(k) \int_{\mathbb{T}} \frac{e^{-|t| k^{2}}}{i \tau+k^{2}} \Phi_{\geq 2 N^{3}}(\tau) v(\tau) d \tau .
\end{aligned}
$$

The contribution of the second term is easily controlled by the first term of the right-hand side of (3.10) since, according to [15],

$$
\sum_{L}\left\langle L+N^{2}\right\rangle^{1 / 2}\left\|\varphi_{N}(k) P_{L}\left(\eta(t) e^{-|t| k^{2}}\right)\right\|_{L_{t}^{2}} \lesssim 1
$$

To treat the contribution of the first one, we set $\theta(t)=\eta(t) e^{(t-|t|) k^{2}}$ and rewrite this contribution as $\sum_{L \leq N^{3}} I_{L}$ with

$$
I_{L}:=\left\langle L+N^{2}\right\rangle^{1 / 2}\left\|\varphi_{N}(k) \varphi_{L}(\tau)\left(\widehat{\theta}\left(\tau^{\prime}\right) \star\left[\Phi_{\geq 2 N^{3}}\left(\tau^{\prime}\right) \frac{v\left(\tau^{\prime}\right)}{i \tau^{\prime}+k^{2}}\right]\right)(\tau)\right\|_{L_{\tau}^{2}} .
$$

For $L \leq N^{3} / 4$, by support considerations we may replace $\hat{\theta}\left(\tau^{\prime}\right)$ by $\chi_{\left|\tau^{\prime}\right| \geq \frac{N^{3}}{2}} \widehat{\theta}\left(\tau^{\prime}\right)$ in (3.11). Since it is not too hard to check that two integrations by parts yield $|\hat{\theta}(\tau)| \lesssim \frac{\langle k\rangle^{2}}{|\tau|^{2}}$, this ensures that

$$
\begin{aligned}
\sum_{L \leq N^{3} / 4} I_{L} & \lesssim \sum_{L \leq N^{3} / 4}\left\langle L+N^{2}\right\rangle^{1 / 2} \varphi_{N}(k)\left\|\chi_{|\tau| \geq \frac{N^{3}}{2}} \widehat{\theta}\right\|_{L^{2}}\left\|\frac{v}{\left\langle i \tau+k^{2}\right\rangle}\right\|_{L^{1}} \\
& \lesssim \sum_{L \leq N^{3} / 4}\left\langle L+N^{2}\right\rangle^{1 / 2} N^{-5 / 2} \varphi_{N}(k)\left\|\frac{v}{\left\langle i \tau+k^{2}\right\rangle}\right\|_{L^{1}} \\
& \lesssim \varphi_{N}(k)\left\|\frac{v}{\left\langle i \tau+k^{2}\right\rangle}\right\|_{L^{1}} .
\end{aligned}
$$

Now, for $L=N^{3}$ (note that the case $L=N^{3} / 2$ can be treated in exactly the same way), we use that $\varphi_{L} \equiv \eta(\cdot / 2 L) \varphi_{L}$ and that by the mean value theorem, $\left|\varphi_{L}(\tau)-\varphi_{L}\left(\tau^{\prime}\right)\right| \lesssim L^{-1}\left|\tau-\tau^{\prime}\right|$. Substituting this in (3.11) we infer that

$$
\begin{aligned}
I_{N^{3}} \lesssim & \left\langle N^{3}+N^{2}\right\rangle^{1 / 2} \varphi_{N}(k)\left\|\int_{\mathbb{R}} \widehat{\theta}\left(\tau-\tau^{\prime}\right) \varphi_{N^{3}}\left(\tau^{\prime}\right) \frac{\Phi_{\geq 2 N^{3}}\left(\tau^{\prime}\right) v\left(\tau^{\prime}\right)}{i \tau^{\prime}+k^{2}} d \tau^{\prime}\right\|_{L_{\tau}^{2}} \\
& +\left\langle N^{3}+N^{2}\right\rangle^{1 / 2} \varphi_{N}(k)\left\|\eta ( \tau / 4 N ^ { 3 } ) N ^ { - 3 } \int _ { \mathbb { R } } \left|\widehat{\theta}\left(\tau-\tau^{\prime}\right)\left\|\tau-\tau^{\prime} \mid \frac{\left|v\left(\tau^{\prime}\right)\right|}{\left|i \tau^{\prime}+k^{2}\right|} d \tau^{\prime}\right\|_{L_{\tau}^{2}}\right.\right. \\
:= & I_{N^{3}}^{1}+I_{N^{3}}^{2} .
\end{aligned}
$$


Applying the Plancherel theorem, the Hölder inequality in $t$ and then the Parseval theorem, the first term can be easily estimated by

$$
\begin{aligned}
I_{N^{3}}^{1} & \lesssim\left\langle N^{3}+N^{2}\right\rangle^{1 / 2} \varphi_{N}(k)\|\theta\|_{L^{\infty}}\left\|\frac{\varphi_{N^{3}}(\tau) v(\tau)}{i \tau+k^{2}}\right\|_{L^{2}} \\
& \lesssim\left\langle N^{3}\right\rangle^{-1 / 2} \varphi_{N}(k)\left\|\varphi_{N^{3}}(\tau) v(\tau)\right\|_{L^{2}}
\end{aligned}
$$

which is acceptable. Finally, note that $\|\widehat{\theta}\|_{L^{\infty}} \leq\|\theta\|_{L^{1}} \leq\|\eta\|_{L^{1}} \lesssim 1$ and that integrating by parts one time, it is not too hard to check that $|\widehat{\theta}(\tau)| \lesssim \frac{1}{\langle\tau\rangle}$. This ensures that the second term can be controlled by

$$
\begin{aligned}
I_{N^{3}} & \lesssim\left\langle N^{3}\right\rangle^{1 / 2} \varphi_{N}(k)\left\|\eta\left(\tau / 4 N^{3}\right) N^{-3} \int_{\mathbb{R}} \frac{\left|\tau-\tau^{\prime}\right|}{\left\langle\tau-\tau^{\prime}\right\rangle} \frac{\left|v\left(\tau^{\prime}\right)\right|}{\left|i \tau^{\prime}+k^{2}\right|} d \tau^{\prime}\right\|_{L^{2}} \\
& \lesssim N^{-3 / 2} \varphi_{N}(k)\left\|\eta\left(\tau / 4 N^{3}\right)\right\|_{L_{\tau}^{2}}\left\|\frac{v(\tau)}{i \tau+k^{2}}\right\|_{L_{\tau}^{1}} \\
& \lesssim \varphi_{N}(k)\left\|\frac{v(\tau)}{i \tau+k^{2}}\right\|_{L_{\tau}^{1}} .
\end{aligned}
$$

\section{Bilinear estimate}

In this section we provide a proof of the following crucial bilinear estimate.

Proposition 4.1. Let $0<\varepsilon<1 / 12$. Then for all $u, v \in \mathcal{S}_{\varepsilon}^{-1}$ it holds

$$
\left\|\partial_{x}(u v)\right\|_{\mathcal{N}_{\varepsilon}^{-1}} \lesssim\|u\|_{\mathcal{S}_{\varepsilon}^{-1}}\|v\|_{\mathcal{S}_{\varepsilon}^{-1}}
$$

We will need the following sharp estimates proved in [18].

Lemma 4.1. Let $u_{1}$ and $u_{2}$ be two real-valued $L^{2}$ functions defined on $\mathbb{R} \times \mathbb{Z}$ with the following support properties:

$$
(\tau, k) \in \operatorname{supp} u_{i} \Rightarrow|k| \sim N_{i},\left\langle\tau-k^{3}\right\rangle \sim L_{i}, i=1,2 .
$$

Then the following estimates hold:

$$
\left\|u_{1} \star u_{2}\right\|_{L_{\tau}^{2} L^{2}(|k| \geq N)} \lesssim \min \left(L_{1}, L_{2}\right)^{1 / 2}\left(\frac{\max \left(L_{1}, L_{2}\right)^{1 / 4}}{N^{1 / 4}}+1\right)\left\|u_{1}\right\|_{L^{2}}\left\|u_{2}\right\|_{L^{2}},
$$

and if $N_{1} \gg N_{2}$,

$$
\left\|u_{1} \star u_{2}\right\|_{L^{2}} \lesssim \min \left(L_{1}, L_{2}\right)^{1 / 2}\left(\frac{\max \left(L_{1}, L_{2}\right)^{1 / 2}}{N_{1}}+1\right)\left\|u_{1}\right\|_{L^{2}}\left\|u_{2}\right\|_{L^{2}} .
$$

Proof of Proposition 4.1. First we remark that because of the $L_{k}^{2}$ structure of the spaces involved in our analysis, we have the following localization property:

$$
\|f\|_{\mathcal{S}_{\varepsilon}^{-1}} \sim\left(\sum_{N}\left\|P_{N} f\right\|_{\mathcal{S}_{\varepsilon}^{-1}}^{2}\right)^{1 / 2} \text { and }\|f\|_{\mathcal{N}_{\varepsilon}^{-1}} \sim\left(\sum_{N}\left\|P_{N} f\right\|_{\mathcal{N}_{\varepsilon}^{-1}}^{2}\right)^{1 / 2}
$$

Performing a dyadic decomposition for $u, v$ we thus obtain

$$
\left\|\partial_{x}(u v)\right\|_{\mathcal{N}_{\varepsilon}^{-1}} \sim\left(\sum_{N}\left\|\sum_{N_{1}, N_{2}} P_{N} \partial_{x}\left(P_{N_{1}} u P_{N_{2}} v\right)\right\|_{\mathcal{N}_{\varepsilon}^{-1}}^{2}\right)^{1 / 2} .
$$


We can now reduce the number of cases to analyze by noting that the right-hand side vanishes unless one of the following cases holds:

- (high-low interaction) $N \sim N_{2}$ and $N_{1} \lesssim N$,

- (low-high interaction) $N \sim N_{1}$ and $N_{2} \lesssim N$,

- (high-high interaction) $N \ll N_{1} \sim N_{2}$.

The two former cases are symmetric. In the first case, we can rewrite the righthand side of (4.2) as

$$
\left\|\partial_{x}(u v)\right\|_{\mathcal{N}_{\varepsilon}^{-1}} \sim\left(\sum_{N}\left\|P_{N} \partial_{x}\left(P_{\lesssim N} u P_{N} v\right)\right\|_{\mathcal{N}_{\varepsilon}^{-1}}^{2}\right)^{1 / 2}
$$

and it suffices to prove the high-low estimate

$$
\left\|P_{N} \partial_{x}\left(P_{\lesssim N} u P_{N} v\right)\right\|_{\mathcal{N}_{\varepsilon}^{-1}} \lesssim\|u\|_{\mathcal{S}_{\varepsilon}^{-1}}\left\|P_{N} v\right\|_{\mathcal{S}_{\varepsilon}^{-1}}
$$

for any dyadic $N$. If we now consider the third case, we easily get

$$
\left\|\partial_{x}(u v)\right\|_{\mathcal{N}_{\varepsilon}^{-1}} \lesssim \sum_{N_{1}}\left\|P_{\ll N_{1}} \partial_{x}\left(P_{N_{1}} u P_{N_{1}} v\right)\right\|_{\mathcal{N}_{\varepsilon}^{-1}},
$$

and it suffices to prove for any $N_{1}$ the high-high estimate

$$
\left\|P_{\ll N_{1}} \partial_{x}\left(P_{N_{1}} u P_{N_{1}} v\right)\right\|_{\mathcal{N}_{\varepsilon}^{-1}} \lesssim\left\|P_{N_{1}} u\right\|_{\mathcal{S}_{\varepsilon}^{-1}}\left\|P_{N_{1}} v\right\|_{\mathcal{S}_{\varepsilon}^{-1}},
$$

since the claim then follows from Cauchy-Schwarz.

Finally, since the $\mathcal{S}_{\varepsilon}^{-1}$-norm only sees the size of the modulus of the spacetime Fourier transform we can always assume that our functions have real-valued nonnegative space-time Fourier transform.

Before starting to estimate the different terms we recall the resonance relation associated with the $\mathrm{KdV}$ equation that reads

$\left(\tau_{1}-k_{1}^{3}\right)+\left(\tau_{2}-k_{2}^{3}\right)+\left(\tau_{3}-k_{3}^{3}\right)=3 k_{1} k_{2} k_{3}$ whenever $\left(\tau_{1}, k_{1}\right)+\left(\tau_{2}, k_{2}\right)+\left(\tau_{3}, k_{3}\right)=0$.

4.1. High-low interactions. We decompose the bilinear term as

$$
P_{N} \partial_{x}\left(P_{\lesssim N} u P_{N} v\right)=\sum_{N_{1} \lesssim N} \sum_{L, L_{1}, L_{2}} P_{N} Q_{L} \partial_{x}\left(P_{N_{1}} Q_{L_{1}} u P_{N} Q_{L_{2}} v\right) .
$$

Note first that we can always assume that $N_{1} \gg 1$, since otherwise, by using Sobolev inequalities and (2.7), it holds

$$
\begin{aligned}
\sum_{N_{1} \lesssim 1}\left\|P_{N} \partial_{x}\left(P_{N_{1}} u P_{N} v\right)\right\|_{Y^{-1,-\frac{1}{2}}} & \lesssim \sum_{N_{1} \lesssim 1}\langle N\rangle^{-1} N\left\|P_{N}\left(P_{N_{1}} u P_{N} v\right)\right\|_{L_{t}^{1} L_{x}^{2}} \\
& \lesssim \sum_{N_{1} \lesssim 1}\left\|P_{N_{1}} u\right\|_{L_{t}^{2} L_{x}^{\infty}}\left\|P_{N} v\right\|_{L^{2}} \\
& \lesssim \sum_{N_{1} \lesssim 1} N_{1}^{1 / 2}\left\|P_{N_{1}} u\right\|_{L^{2}}\left\|P_{N} v\right\|_{L^{2}} \\
& \lesssim\|u\|_{\mathcal{S}_{\varepsilon}^{-1}}\left\|P_{N} v\right\|_{\mathcal{S}_{\varepsilon}^{-1}}
\end{aligned}
$$

as soon as $\varepsilon \leq 1 / 2$. We now separate different regions. It is worth noticing that (4.3) ensures that $\max \left(L, L_{1}, L_{2}\right) \gtrsim N^{2} N_{1}$. 
4.1.1. $L \gtrsim N^{2} N_{1}$. We set $L \sim 2^{l} N^{2} N_{1}$. Taking advantage of the $X_{\varepsilon}^{-1,-\frac{1}{2}, 1} \cap Z^{-1,-\frac{1}{2}}$ part of $\mathcal{N}_{\varepsilon}^{-1}$ as well as the continuous embedding $X^{-1,-\frac{1}{2}, 1} \hookrightarrow X_{\varepsilon}^{-1,-\frac{1}{2}, 1} \cap Z^{-1,-\frac{1}{2}}$, by using Lemma 4.1 we get

$$
\begin{aligned}
I_{1}: & =\sum_{1 \ll N_{1} \lesssim N} \sum_{\substack{l \geq 0 \\
m y L_{1}, L_{2}}}\left\|P_{N} Q_{L} \partial_{x}\left(P_{N_{1}} Q_{L_{1}} u P_{N} Q_{L_{2}} v\right)\right\|_{X^{-1,-\frac{1}{2}, 1}} \\
& \lesssim \sum_{1 \ll N_{1} \lesssim N} \sum_{\substack{l \geq 0 \\
m y L_{1}, L_{2}}} 2^{-l / 2} N^{-1} N_{1}^{-1 / 2}\left\|P_{N} Q_{L}\left(P_{N_{1}} Q_{L_{1}} u P_{N} Q_{L_{2}} v\right)\right\|_{L^{2}} \\
& \lesssim \sum_{1 \ll N_{1} \lesssim N} \sum_{\substack{l \geq 0 \\
m y L_{1}, L_{2}}} 2^{-l / 2} N^{-1} N_{1}^{-1 / 2}\left(L_{1} \wedge L_{2}\right)^{1 / 2}\left(\frac{\left(L_{1} \vee L_{2}\right)^{1 / 4}}{N^{1 / 4}}+1\right) \\
& \times\left\|P_{N_{1}} Q_{L_{1}} u\right\|_{L^{2}}\left\|P_{N} Q_{L_{2}} v\right\|_{L^{2}} .
\end{aligned}
$$

Noticing that for any $0<\alpha<1$ it holds

$$
\left(L_{1} \wedge L_{2}\right)^{1 / 2}\left(\frac{\left(L_{1} \vee L_{2}\right)^{1 / 4}}{N^{1 / 4}}+1\right) \lesssim\left(L_{1} \vee L_{2}\right)^{-\frac{\alpha}{4}} N^{-\frac{3-2 \alpha}{4}}\left(L_{1}+N_{1}^{2}\right)^{1 / 2}\left(L_{2}+N^{2}\right)^{1 / 2},
$$

we deduce that

$$
\begin{aligned}
I_{1} \lesssim & \sum_{1 \ll N_{1} \lesssim N} \sum_{\substack{l \geq 0 \\
m y \bar{L}_{1}, L_{2}}} 2^{-l / 2}\left(L_{1} L_{2}\right)^{-\alpha / 8}\left(\frac{N_{1}}{N}\right)^{\frac{5-2 \alpha}{8}} \\
& \times\left\|P_{N_{1}} Q_{L_{1}} u\right\|_{X^{-\frac{9-2 \alpha}{8}}, \frac{1}{2}, 1}\left\|P_{N} Q_{L_{2}} v\right\|_{X^{-\frac{9-2 \alpha}{8}, \frac{1}{2}, 1}}
\end{aligned}
$$

Taking $\alpha>0$ small enough, this proves with (2.8) that

$$
I_{1} \lesssim\|u\|_{\mathcal{S}_{\varepsilon}^{-1}}\left\|P_{N} v\right\|_{\mathcal{S}_{\varepsilon}^{-1}}
$$

whenever $\varepsilon<1 / 8$.

4.1.2. $L_{1} \gtrsim N^{2} N_{1}$ and $L \ll N^{2} N_{1}$. We can set $L_{1} \sim 2^{l} N^{2} N_{1}$ with $l \geq 0$. By duality, it is equivalent to show that 1

$$
I_{2} \lesssim\|u\|_{\mathcal{S}_{\varepsilon}^{-1}}\left\|P_{N} v\right\|_{\mathcal{S}_{\varepsilon}^{-1}}\left\|P_{N} w\right\|_{X^{1, \frac{1}{2}, \infty}}
$$

where

$$
\begin{aligned}
I_{2} & :=\sum_{1 \ll N_{1} \lesssim N} \sum_{\substack{l \geq 0 \\
m y L_{2}, L \ll N^{2} N_{1}}}\left|\left(P_{N} Q_{L} w, \partial_{x}\left(P_{N_{1}} Q_{2^{l} N^{2} N_{1}} u P_{N} Q_{L_{2}} v\right)\right)_{L^{2}}\right| \\
& =\sum_{1 \ll N_{1} \lesssim N} \sum_{\substack{l \geq 0 \\
m y L_{2}, L \ll N^{2} N_{1}}}\left|\left(P_{N_{1}} \widetilde{Q_{2^{l} N^{2} N_{1}}} u, \widetilde{\partial_{x}} \widetilde{P_{N} Q_{L}} w \star \widetilde{P_{N} Q_{L_{2}}} v\right)_{L^{2}}\right|
\end{aligned}
$$

${ }^{1}$ The space $X^{1, \frac{1}{2}, \infty}$ is endowed with the norm

$$
\|u\|_{X^{1, \frac{1}{2}, \infty}}:=\left(\sum_{N} \sup _{L}\left[\langle N\rangle\left\langle L+N^{2}\right\rangle^{\frac{1}{2}}\left\|P_{N} Q_{L} u\right\|_{L_{x t}^{2}}\right]^{2}\right)^{1 / 2} .
$$


and $\check{\theta}(\tau, k)=\theta(-\tau,-k)$. According to Lemma 4.1 we get

$$
\begin{aligned}
I_{2} \lesssim & \sum_{1 \ll N_{1} \lesssim N} \sum_{\substack{l \geq 0 \\
m y L_{2}, L \lesssim N^{2} N_{1}}} 2^{-l / 2} N^{-1} N_{1}^{-1 / 2}\left(L_{1}^{1 / 2}\left\|P_{N_{1}} Q_{2^{l} N^{2} N_{1}} u\right\|_{L^{2}}\right) \\
& \times \|{ }_{\partial_{x} \widehat{P_{N} Q_{L}} w \star \widehat{P_{N} Q_{L_{2}}} v \|_{L^{2}}} \sum_{\substack { 1 \ll N_{1} \lesssim N \\
\begin{subarray}{c}{l \geq 0 \\
m y L_{2}, L \lesssim N^{2} N_{1}{ 1 \ll N _ { 1 } \lesssim N \\
\begin{subarray} { c } { l \geq 0 \\
m y L _ { 2 } , L \lesssim N ^ { 2 } N _ { 1 } } }\end{subarray}} 2^{-l / 2} N^{-1} N_{1}^{-1 / 2}\left(L_{1}^{1 / 2}\left\|P_{N_{1}} Q_{2^{l} N^{2} N_{1}} u\right\|_{L^{2}}\right) \\
& \times\left(L \wedge L_{2}\right)^{1 / 2}\left(\frac{\left(L \vee L_{2}\right)^{1 / 4}}{N_{1}^{1 / 4}}+1\right)\left\|\partial_{x} P_{N} Q_{L} w\right\|_{L^{2}}\left\|P_{N} Q_{L_{2}} v\right\|_{L^{2}} .
\end{aligned}
$$

Since for any $\varepsilon>0$ we have the estimate

$$
\left(L \wedge L_{2}\right)^{1 / 2}\left(\frac{\left(L \vee L_{2}\right)^{1 / 4}}{N_{1}^{1 / 4}}+1\right) \lesssim\left(L \vee L_{2}\right)^{-\varepsilon / 2} N_{1}^{-\frac{3}{4}+\varepsilon}\left(L+N^{2}\right)^{1 / 2}\left(L_{2}+N^{2}\right)^{1 / 2},
$$

it follows that

$$
\begin{aligned}
I_{2} \lesssim & \sum_{1 \ll N_{1} \lesssim N} \sum_{\substack{l \geq 0 \\
m y L_{2}, L \lesssim N^{2} N_{1}}} 2^{-l / 2}\left(L L_{2}\right)^{-\varepsilon / 4} N_{1}^{-\frac{1}{4}+3 \varepsilon} \\
& \times\left\|P_{N_{1}} Q_{2^{l} N^{2} N_{1}} u\right\|_{X^{-1-\varepsilon, \frac{1}{2}, 1}}\left\|P_{N} Q_{L} w\right\|_{X^{1, \frac{1}{2}, \infty}}\left\|P_{N} Q_{L_{2}} v\right\|_{X^{-1-\varepsilon, \frac{1}{2}, 1}},
\end{aligned}
$$

which is acceptable whenever $\varepsilon<1 / 12$.

4.1.3. $L_{2} \gtrsim N^{2} N_{1}$ and $L \vee L_{1} \ll N^{2} N_{1}$. In this region, thanks to the resonance relation (4.3), one has $L_{2} \sim N^{2} N_{1}$. We proceed as in the preceding subsection. We get

$$
\begin{aligned}
I_{3} & :=\sum_{1 \ll N_{1} \lesssim N} \sum_{L \vee L_{1} \ll N^{2} N_{1}}\left|\left(P_{N} Q_{L} w, \partial_{x}\left(P_{N} Q_{N^{2} N_{1}} v P_{N_{1}} Q_{L_{1}} u\right)\right)_{L^{2}}\right| \\
& \lesssim \sum_{1 \ll N_{1} \lesssim N} \sum_{L \vee L_{1} \lesssim N^{2} N_{1}} N^{-1} N_{1}^{-1 / 2}\left(L_{2}^{1 / 2}\left\|P_{N} Q_{N^{2} N_{1}} v\right\|_{L^{2}}\right) \\
& \times\left(L \wedge L_{1}\right)^{1 / 2}\left(\frac{\left(L \vee L_{1}\right)^{1 / 4}}{N^{1 / 4}}+1\right)\left\|\partial_{x} P_{N} Q_{L} w\right\|_{L^{2}}\left\|P_{N_{1}} Q_{L_{1}} u\right\|_{L^{2}} .
\end{aligned}
$$

On the other hand, we clearly have

$$
\left(L \wedge L_{1}\right)^{1 / 2}\left(\frac{\left(L \vee L_{1}\right)^{1 / 4}}{N^{1 / 4}}+1\right) \lesssim\left(L \vee L_{1}\right)^{-\varepsilon / 2} N_{1}^{-\frac{3}{4}+\varepsilon}\left(L+N^{2}\right)^{1 / 2}\left(L_{1}+N_{1}^{2}\right)^{1 / 2}
$$

Inserting this into (4.5) we deduce

$$
\begin{aligned}
I_{3} \lesssim & \sum_{\substack{1 \ll N_{1} \lesssim N\\
}} \sum_{L \vee L_{1} \lesssim N^{2} N_{1}}\left(L L_{1}\right)^{-\varepsilon / 4} N_{1}^{-\frac{1}{4}+2 \varepsilon}\left\|P_{N} Q_{N^{2} N_{1}} v\right\|_{X^{-1, \frac{1}{2}, 1}} \\
& \times\left\|P_{N} Q_{L} w\right\|_{X^{1, \frac{1}{2}, \infty}}\left\|P_{N_{1}} Q_{L_{1}} u\right\|_{X^{-1-\varepsilon, \frac{1}{2}, 1}} .
\end{aligned}
$$

Now either $N_{1} \leq N$ or $N_{1} \sim N$. In the first case we have $\left\|P_{N} Q_{N^{2} N_{1}} v\right\|_{X^{-1, \frac{1}{2}, 1}}=$ $\left\|P_{N} Q_{N^{2} N_{1}} v\right\|_{X_{\varepsilon}^{-1, \frac{1}{2}, 1}}$, which shows that (4.6) is acceptable for $0<\varepsilon<1 / 8$. In the second case, we have $N_{1}^{-\varepsilon}\left\|P_{N} Q_{N^{2} N_{1}} v\right\|_{X^{-1, \frac{1}{2}, 1}} \leq\left\|P_{N} Q_{N^{2} N_{1}} v\right\|_{X_{\varepsilon}^{-1, \frac{1}{2}, 1}}$, which shows that (4.6) is acceptable for $0<\varepsilon<1 / 12$. 
4.2. High-high interactions. We perform the decomposition

$$
P_{\ll N_{1}} \partial_{x}\left(P_{N_{1}} u P_{N_{1}} v\right)=\sum_{N \ll N_{1}} \sum_{L, L_{1}, L_{2}} P_{N} Q_{L} \partial_{x}\left(P_{N_{1}} Q_{L_{1}} u P_{N_{1}} Q_{L_{2}} v\right) .
$$

By symmetry we can assume that $L_{1} \geq L_{2}$. Then (4.3) ensures that $\max \left(L, L_{1}\right) \gtrsim$ $N N_{1}^{2}$.

4.2.1. $N_{1}^{2} N \lesssim L_{1} \leq N_{1}^{3}$. We can set $L_{1} \sim 2^{l} N_{1}^{2} N$ with $l \geq 0$. Using the $Y^{-1,-\frac{1}{2}}$ part of $\mathcal{N}_{\varepsilon}^{-1}$, we want to estimate

$$
\begin{aligned}
I_{4} & :=\left\|\sum_{\substack{N \ll N_{1} \\
l \geq 0}} P_{N} \partial_{x}\left(P_{N_{1}} Q_{2^{l} N_{1}^{2} N} u P_{N_{1}} v\right)\right\|_{Y^{-1,-\frac{1}{2}}} \\
& \lesssim\left(\sum_{\substack{N \ll N_{1} \\
l \geq 0}}\left\|P_{N}\left(P_{N_{1}} Q_{2^{l} N_{1}^{2} N} u P_{N_{1}} v\right)\right\|_{L_{t}^{1} L_{x}^{2}}^{2}\right)^{1 / 2} \\
& \lesssim\left(\sum_{\substack{N \ll N_{1} \\
l \geq 0}}\left[N^{1 / 2}\left\|P_{N_{1}} Q_{2^{l} N_{1}^{2} N} u\right\|_{L^{2}}\left\|P_{N_{1}} v\right\|_{L^{2}}\right]^{2}\right)^{1 / 2} .
\end{aligned}
$$

According to (2.7) and (2.8), this leads for $\varepsilon \leq 1 / 2$ to

$$
\begin{aligned}
I_{4} & \lesssim\left(\sum_{\substack{N \ll N_{1} \\
l \geq 0}} 2^{-l} \sum_{L_{1} \sim 2^{l} N_{1}^{2} N \leq N_{1}^{3}}\left[N_{1}^{-1} L_{1}^{1 / 2}\left\|P_{N_{1}} Q_{L_{1}} u\right\|_{L^{2}}\right]^{2}\right)^{1 / 2}\left\|P_{N_{1}} v\right\|_{\mathcal{S}_{\varepsilon}^{-1}} \\
& \lesssim\left(\sum_{\substack{L_{1} \leq N_{1}^{3} \\
l \geq 0}} 2^{-l} \sum_{\substack{N \sim 2^{-l} L_{1} N_{1}^{-2}\\
}}\left[N_{1}^{-1} L_{1}^{1 / 2}\left\|P_{N_{1}} Q_{L_{1}} u\right\|_{L^{2}}\right]^{2}\right)^{1 / 2}\left\|P_{N_{1}} v\right\|_{\mathcal{S}_{\varepsilon}^{-1}} \\
& \lesssim\left(\sum_{\substack{L_{1} \leq N_{1}^{3}\\
}}\left[N_{1}^{-1} L_{1}^{1 / 2}\left\|P_{N_{1}} Q_{L_{1}} u\right\|_{L^{2}}\right]^{2}\right)^{1 / 2}\left\|P_{N_{1}} v\right\|_{\mathcal{S}_{\varepsilon}^{-1}} \\
& \lesssim\left\|P_{N_{1}} u\right\|_{\mathcal{S}_{\varepsilon}^{-1}}\left\|P_{N_{1}} v\right\|_{\mathcal{S}_{\varepsilon}^{-1}} .
\end{aligned}
$$

4.2.2. $L_{1} \geq N_{1}^{3}$. We can set $L_{1}=2^{l} N_{1}^{3}$ with $l \geq 0$. We proceed by duality as in Subsection 4.1 .2 to get

$$
\begin{aligned}
& I_{5} \lesssim \sum_{1 \lesssim N \ll N_{1}} \sum_{\substack{l \geq 0 \\
m y L_{2}, L}} 2^{-l / 2} N_{1}^{-\frac{1}{2}+\varepsilon}\left(N_{1}^{-1-\varepsilon} L_{1}^{1 / 2}\left\|P_{N_{1}} Q_{2^{l} N_{1}^{3}} u\right\|_{L^{2}}\right) \\
& \times\left\|\partial_{x} \widetilde{P_{N} Q_{L}} w \star \widetilde{P_{N_{1} Q_{L_{2}}}} v\right\|_{L^{2}} .
\end{aligned}
$$

By virtue of Lemma 4.1, we have the bound

$$
\begin{aligned}
\| \partial_{x} & \widetilde{P_{N} Q_{L}} w \star \widetilde{P_{N_{1}} Q_{L_{2}}} v \|_{L^{2}} \\
& \lesssim\left(L \wedge L_{2}\right)^{1 / 2}\left(\frac{\left(L \vee L_{2}\right)^{1 / 4}}{N_{1}^{1 / 4}}+1\right)\left\|\partial_{x} P_{N} Q_{L} w\right\|_{L^{2}}\left\|P_{N_{1}} Q_{L_{2}} v\right\|_{L^{2}} \\
& \lesssim\left(L \vee L_{2}\right)^{-\varepsilon / 2} N_{1}^{\frac{1}{4}+2 \varepsilon}\left\|P_{N} Q_{L} w\right\|_{X^{1, \frac{1}{2}, \infty}}\left\|P_{N_{1}} Q_{L_{2}} v\right\|_{X^{-1-\varepsilon, \frac{1}{2}, 1}}
\end{aligned}
$$


Thus it is enough to check that

$$
\sum_{1 \lesssim N \ll N_{1}} \sum_{\substack{l \geq 0 \\ m y \bar{L}_{2}, L}} 2^{-l / 2}\left(L L_{2}\right)^{-\varepsilon / 4} N_{1}^{-\frac{1}{4}+3 \varepsilon} \lesssim 1,
$$

but this is easily verified for $\varepsilon<1 / 12$.

4.2.3. $L \gtrsim N_{1}^{2} N$ and $N_{1}^{2} N^{1-\varepsilon} \leq L_{2} \leq L_{1} \ll N_{1}^{2} N$. Then, by the resonance relation (4.3) we must have $L \sim N_{1}^{2} N$. We set $L_{2} \sim 2^{q} N_{1}^{2} N^{1-\varepsilon}$ and $L_{1}=2^{p} L_{2}$ with $q \geq 0$ and $p \geq 0$. Since $N \ll N_{1}$ we are in the region $L \gtrsim N^{3}$. However, since $X^{-1,-\frac{1}{2}, 1} \hookrightarrow X^{-1-\varepsilon,-\frac{1}{2}, 1} \cap Z^{-1,-\frac{1}{2}}$, it suffices to show that

$$
I_{6} \lesssim\left\|P_{N_{1}} u\right\|_{\mathcal{S}_{\varepsilon}^{-1}}\left\|P_{N_{1}} v\right\|_{\mathcal{S}_{\varepsilon}^{-1}}\left(\sum_{N}\left\|P_{N} Q_{N_{1}^{2} N} w\right\|_{X^{1, \frac{1}{2}, \infty}}^{2}\right)^{1 / 2}
$$

where

$$
I_{6}:=\sum_{N \ll N_{1}} \sum_{p \geq 0, q \geq 0}\left|\left(P_{N} Q_{N_{1}^{2} N} w, \partial_{x}\left(P_{N_{1}} Q_{L_{1}} u P_{N_{1}} Q_{L_{2}} v\right)\right)_{L^{2}}\right| .
$$

Using Lemma 4.1 we get

$$
\begin{aligned}
I_{6} \lesssim & \sum_{N \ll N_{1}} \sum_{p \geq 0, q \geq 0}\left\|P_{N_{1}} Q_{L_{1}} u\right\|_{L^{2}} N_{1}^{-1}\left\|P_{N} Q_{N_{1}^{2} N} w\right\|_{X^{1, \frac{1}{2}, \infty}}\left\|P_{N_{1}} Q_{L_{2}} v\right\|_{X^{0, \frac{1}{2}, 1}} \\
\lesssim & \sum_{N \ll N_{1}} \sum_{p \geq 0, q \geq 0} 2^{-q / 2} 2^{-p / 2} N^{-\frac{1}{2}+\frac{\varepsilon}{2}}\left\|P_{N_{1}} Q_{2^{p} 2^{q} N_{1}^{2} N^{1-\varepsilon}} Q_{\leq N_{1}^{3}} u\right\|_{X^{-1, \frac{1}{2}, 1}} \\
& \times\left\|P_{N} Q_{N_{1}^{2} N} w\right\|_{X^{1, \frac{1}{2}, \infty}}\left\|P_{N_{1}} Q_{2^{q} N_{1}^{2} N^{1-\varepsilon}} Q_{\leq N_{1}^{3}} v\right\|_{X^{-1, \frac{1}{2}, 1}},
\end{aligned}
$$

which is acceptable as soon as $\varepsilon<1$.

4.2.4. $L \gtrsim N_{1}^{2} N, L_{1} \ll N_{1}^{2} N$ and $L_{2} \leq N_{1}^{2} N^{1-\varepsilon}$. Since $N \ll N_{1}$ we are in the region $L>N^{3}$. It thus suffices to estimate both the $X^{-1-\varepsilon,-\frac{1}{2}, 1}$ and the $Z^{-1,-\frac{1}{2}}$ norms. Let us start by estimating the first norm. Note that in this region we can replace $P_{N_{1}} u$ and $P_{N_{1}} v$ by $P_{N_{1}} Q_{\leq N_{1}^{3}} u$ and $P_{N_{1}} Q_{\leq N_{1}^{3}} v$. Taking into account the gain of $\varepsilon$ in the definition of the space, we get

$$
\begin{aligned}
\left\|\partial_{x}(u v)\right\|_{X^{-1-\varepsilon,-\frac{1}{2}, 1}} & \lesssim \sum_{1 \lesssim N \ll N_{1}} N^{-\varepsilon} N_{1}^{-1} N^{-1 / 2}\left\|P_{N}\left(P_{N_{1}} Q_{\leq N_{1}^{3}} u P_{N_{1}} Q_{\leq N_{1}^{3}} v\right)\right\|_{L^{2}} \\
& \lesssim \sum_{1 \lesssim N \ll N_{1}} N^{-\varepsilon} N_{1}^{-1}\left\|P_{N_{1}} Q_{\leq N_{1}^{3}} u P_{N_{1}} Q_{\leq N_{1}^{3}} v\right\|_{L_{t}^{2} L_{x}^{1}} \\
& \lesssim \sum_{1 \lesssim N \ll N_{1}} N^{-\varepsilon}\left(N_{1}^{-1}\left\|P_{N_{1}} Q_{\leq N_{1}^{3}} u\right\|_{\left.L_{t}^{\infty} L_{x}^{2}\right)\left\|P_{N_{1}} Q_{\leq N_{1}^{3}} v\right\|_{L^{2}}}\right.
\end{aligned}
$$

which is acceptable as soon as $\varepsilon>0$. It remains to estimate the $Z^{-1,-\frac{1}{2}}$-norm. By duality we have to estimate

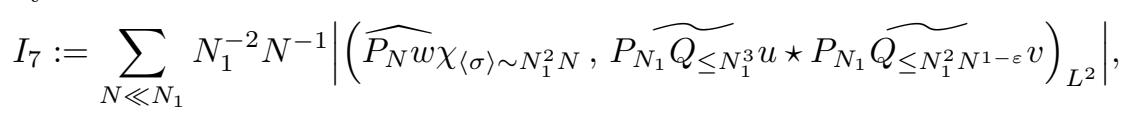

where $w$ only depends on $k$ and with $\sigma=\tau-k^{3}$ (recall that we can assume that the space-time Fourier transforms of $u$ and $w$ are nonnegative real-valued functions). We follow an idea that can be found in 4. First we notice that for any fixed $k$,

$$
\chi_{\langle\sigma\rangle \sim L} \lesssim \chi_{\langle\sigma\rangle \sim L} \star_{\tau}\left(\frac{1}{L} \chi_{\langle\sigma\rangle \leq L}\right)
$$


and thus the above scalar product can be rewritten as

$$
\left|\left(\widehat{P_{N} w} \chi_{\langle\sigma\rangle \sim N_{1}^{2} N}, \widehat{P_{N_{1}} Q_{\leq N_{1}^{3}}} u \star P_{N_{1}} \widetilde{Q_{\leq N_{1}^{2} N^{1-\varepsilon}}} v \star_{\tau}\left(\frac{1}{N_{1}^{2} N} \chi_{\langle\sigma\rangle \leq N_{1}^{2} N}\right)\right)_{L^{2}}\right|,
$$

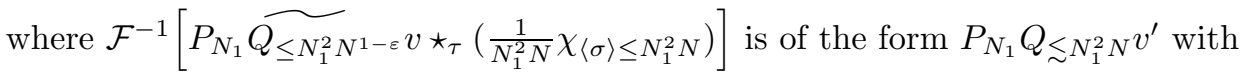

$$
\left\|P_{N_{1}} Q_{\lesssim N_{1}^{2} N} v^{\prime}\right\|_{L^{2}} \lesssim N^{-\varepsilon / 2}\left\|P_{N_{1}} Q_{\leq N_{1}^{2} N^{1-\varepsilon}} v\right\|_{L^{2}} .
$$

Indeed the linear operator $T_{K, K_{2}}: v \mapsto \frac{1}{K} v(\cdot) \chi_{\left\{\langle\cdot\rangle \leq K_{2}\right\}} \star \chi_{\{\langle\cdot\rangle \leq K\}}$ is a continuous endomorphism of $L^{1}(\mathbb{R})$ and $L^{\infty}(\mathbb{R})$ with

$$
\begin{aligned}
\left\|T_{K, K_{2}} v\right\|_{L^{\infty}(\mathbb{R})} & \leq \sup _{x \in \mathbb{R}} \frac{1}{K}\left|\int_{\mathbb{R}} v(y) \chi_{\left\{\langle y\rangle \leq K_{2}\right\}} \chi_{\{\langle x-y\rangle \leq K\}} d y\right| \\
& \lesssim \frac{\min \left(K, K_{2}\right)}{K}\|v\|_{L^{\infty}(\mathbb{R})}
\end{aligned}
$$

and

$$
\left\|T_{K, K_{2}} v\right\|_{L^{1}(\mathbb{R})} \leq \frac{1}{K}\|v\|_{L^{1}(\mathbb{R})}\left\|\chi_{\{\langle\cdot\rangle \leq K\}}\right\|_{L^{1}(\mathbb{R})} \lesssim\|v\|_{L^{1}(\mathbb{R})} .
$$

Therefore, by the Riesz interpolation theorem $T_{K, K_{2}}$ is a continuous endomorphism of $L^{2}(\mathbb{R})$ with

$$
\left\|T_{K, K_{2}} v\right\|_{L^{2}(\mathbb{R})} \lesssim \min \left(1, \frac{K_{2}}{K}\right)^{1 / 2}\|v\|_{L^{2}(\mathbb{R})} .
$$

Hence, by Sobolev in $k$ and (4.7),

$$
\begin{aligned}
I_{7} & \lesssim \sum_{N \ll N_{1}} N_{1}^{-2} N^{-1} N^{1 / 2}\left\|\widehat{P_{N} w} \chi_{\langle\sigma\rangle \sim N_{1}^{2} N}\right\|_{L^{2}}\left\|P_{N_{1}} Q_{\leq N_{1}^{3}} u\right\|_{L_{t}^{\infty} L_{x}^{2}}\left\|P_{N_{1}} Q_{\lesssim N_{1}^{2} N} v^{\prime}\right\|_{L^{2}} \\
& \lesssim \sum_{N \ll N_{1}} N^{-\varepsilon / 2}\left\|P_{N} w\right\|_{L^{2}(\mathbb{T})}\left(N_{1}^{-1}\left\|P_{N_{1}} Q_{\leq N_{1}^{3}} u\right\|_{L_{t}^{\infty} L_{x}^{2}}\right)\left\|P_{N_{1}} v\right\|_{L^{2}},
\end{aligned}
$$

which is acceptable as soon as $\varepsilon>0$.

\section{WELL-POSEDNESS}

In this section, we prove the well-posedness result. The proof follows exactly the same lines as in [15]. Using a standard fixed point procedure, it is clear that the bilinear estimate (4.1) allows us to show local well-posedness, but for small initial data only. This is because $H^{-1}$ appears as a critical space for KdV-Burgers. Indeed, on one hand, we cannot obtain any contraction factor by restricting time. On the other hand, a dilation argument does not work here since the reduction of the $H^{-1}$-norm of the dilated initial data would be exactly compensated by the diminution of the dissipative coefficient in front of $u_{x x}$ (that we take equal to 1 in (1.1)) in the equation satisfied by the dilated solution. In order to remove the size restriction on the data, we change the metric on our resolution space.

For $0<\varepsilon<1 / 12$ and $\beta \geq 1$, let us define the following norm on $\widetilde{\mathcal{S}_{\varepsilon}^{-1}}$ :

$$
\|u\|_{\mathcal{Z}_{\beta}}=\inf _{\substack{u=u_{1}+u_{2} \\ u_{1} \in \widetilde{\mathcal{S}_{\varepsilon}^{-1}}, u_{2} \in \widetilde{\mathcal{S}_{\varepsilon}^{0}}}}\left\{\left\|u_{1}\right\|_{\widetilde{\mathcal{S}_{\varepsilon}^{-1}}}+\frac{1}{\beta}\left\|u_{2}\right\|_{\widetilde{\mathcal{S}_{\varepsilon}^{0}}}\right\} \text {. }
$$

Note that this norm is equivalent to $\|\cdot\|_{\widetilde{\mathcal{S}_{\varepsilon}^{-1}}}$. Now we will need the following modification of Proposition 4.1. This new proposition means that as soon as we assume more regularity on $u$ we can get a contractive factor for small times in the bilinear estimate. 
Proposition 5.1. There exists $\nu>0$ such that for any $0<\varepsilon<1 / 12$ and all $(u, v) \in \mathcal{S}_{\varepsilon}^{0} \times \mathcal{S}_{\varepsilon}^{-1}$, with compact support (in time) in $[-T, T]$, it holds

$$
\left\|\partial_{x}(u v)\right\|_{\mathcal{N}_{\varepsilon}^{-1}} \lesssim T^{\nu}\|u\|_{\mathcal{S}_{\varepsilon}^{0}}\|v\|_{\mathcal{S}_{\varepsilon}^{-1}} .
$$

Proof. It suffices to slightly modify the proof of Proposition 4.1 to make use of the following result that can be found in [12, Lemma 3.1] (see also [16, Lemma 3.6]): For any $\theta>0$, there exists $\mu=\mu(\theta)>0$ such that for any smooth function $f$ with compact support in time in $[-T, T]$,

$$
\left\|\mathcal{F}_{t, x}^{-1}\left(\frac{\hat{f}(\tau, k)}{\left\langle\tau-k^{3}\right\rangle^{\theta}}\right)\right\|_{L_{t, x}^{2}} \lesssim T^{\mu}\|f\|_{L_{t, x}^{2,2}} .
$$

According to (2.8) this ensures, in particular, that for any $w \in \mathcal{S}_{3 / 8}^{0}$ with compact support in $[-T, T]$, it holds

$$
\|w\|_{L_{t}^{2} H^{3 / 4}} \lesssim\|w\|_{X_{3 / 8}^{0, \frac{3}{8}, 2}} \lesssim T^{\mu\left(\frac{1}{8}\right)}\|w\|_{X_{3 / 8}^{0, \frac{1}{2}, 2}} \lesssim T^{\mu\left(\frac{1}{8}\right)}\|w\|_{\mathcal{S}_{3 / 8}^{0}} .
$$

It is pretty clear that the interactions between high frequencies of $u$ and high or low frequencies of $v$ can be treated by following the proof of Proposition 4.1 and using (5.3). The region that seems the most dangerous is the one of interactions between low frequencies of $u$ and high frequencies of $v$ in the proof of Proposition 4.1. But actually in this region, except in the subregion $N_{1} \lesssim 1$, we can notice that we may keep some powers of $L_{1}$ or $L_{2}$ in the estimates, and thus (5.3) ensures that (5.1) holds (one can even replace $\mathcal{S}_{\varepsilon}^{0}$ by $\mathcal{S}_{\varepsilon}^{-1}$ ). Finally, in the subregion $N_{1} \lesssim 1$, (5.1) follows directly by applying (5.3) in the next to the last line in (4.4).

We are now in a position to prove that the application

$$
F_{\phi}^{T}: u \mapsto \eta(t)\left[W(t) \phi-\frac{1}{2} \mathcal{L} \partial_{x}\left(\eta_{T} u\right)^{2}\right],
$$

where $\mathcal{L}$ is defined in (3.2), is contractive on a ball of $\mathcal{Z}_{\beta}$ for a suitable $\beta>0$ and $T>0$ small enough. Assuming this for a while, the local part of Theorem 1.1 follows by using standard arguments. Note that the uniqueness will hold in the restriction spaces $\widetilde{\mathcal{S}_{\varepsilon}^{-1}}(\tau)$ endowed with the norm

$$
\|u\|_{\widetilde{\mathcal{S}_{\varepsilon}^{-1}}(\tau)}:=\inf _{v \in \widetilde{\mathcal{S}_{\varepsilon}^{-1}}}\left\{\|v\|_{\widetilde{\mathcal{S}_{\varepsilon}^{-1}}}, v \equiv u \text { on }[0, \tau]\right\} .
$$

Finally, to see that the solution $u$ can be extended for all positive times and belongs to $C\left(\mathbb{R}_{+}^{*} ; H^{\infty}\right)$, it suffices to notice that, according to (2.7), $u \in \mathcal{S}_{\varepsilon}^{-1}(\tau) \hookrightarrow$ $L^{2}(] 0, \tau[\times \mathbb{T})$. Therefore, for any $0<\tau^{\prime}<\tau$ there exists $\left.t_{0} \in\right] 0, \tau^{\prime}\left[\right.$ such that $u\left(t_{0}\right)$ belongs to $L^{2}(\mathbb{T})$. Since according to [16, (1.1) is globally well-posed in $L^{2}(\mathbb{T})$ with a solution belonging to $C\left(\mathbb{R}_{+}^{*} ; H^{\infty}(\mathbb{T})\right)$, the conclusion follows.

In order to prove that $F_{\phi}^{T}$ is contractive, the first step is to establish the following result.

Proposition 5.2. For any $\beta \geq 1$ there exists $0<T=T(\beta)<1$ such that for any $u, v \in \mathcal{Z}_{\beta}$ with compact support in $[-T, T]$, we have

$$
\left\|\mathcal{L} \partial_{x}(u v)\right\|_{\mathcal{Z}_{\beta}} \lesssim\|u\|_{\mathcal{Z}_{\beta}}\|v\|_{\mathcal{Z}_{\beta}} .
$$


Assume for the moment that (5.4) holds and let $u_{0} \in H^{-1}$ and $\alpha>0$. Split the data $u_{0}$ into low and high frequencies:

$$
u_{0}=P_{\lesssim N} u_{0}+P_{\gg N} u_{0}
$$

for a dyadic number $N$. Taking $N=N(\alpha)$ large enough, it is obvious to check that $\left\|P_{\gg N} u_{0}\right\|_{H^{-1}} \leq \alpha$. Hence, according to (3.1),

$$
\left\|\eta(\cdot) W(\cdot) P_{\gg N} u_{0}\right\|_{\mathcal{Z}_{\beta}} \lesssim \alpha .
$$

Now using the $\widetilde{\mathcal{S}_{\varepsilon}^{0}}$-part of $\mathcal{Z}_{\beta}$, we control the low frequencies as follows:

$$
\left\|\eta(\cdot) W(\cdot) P_{\lesssim N} u_{0}\right\|_{\widetilde{\mathcal{S}_{\varepsilon}^{0}}} \lesssim \frac{1}{\beta}\left\|P_{\lesssim N} u_{0}\right\|_{L^{2}} \lesssim \frac{N}{\beta}\left\|u_{0}\right\|_{H^{-1}} .
$$

Thus we get

$$
\left\|\eta(\cdot) W(\cdot) P_{\lesssim N} u_{0}\right\|_{\mathcal{Z}_{\beta}} \lesssim \alpha \text { for } \beta \gtrsim \frac{N\left\|u_{0}\right\|_{H^{-1}}}{\alpha}
$$

Since $\alpha$ can be chosen as small as needed, we conclude with (5.4) that $F_{\phi}^{T}$ is contractive on a ball of $\mathcal{Z}_{\beta}$ of radius $R \sim \alpha$ as soon as $\beta \gtrsim N\left\|u_{0}\right\|_{H^{-1}} / \alpha$ and $T=T(\beta)$.

Proof of Proposition 5.2. By definition on the function space $\mathcal{Z}_{\beta}$, there exist $u_{1}, v_{1}$ $\in \widetilde{\mathcal{S}_{\varepsilon}^{-1}}$ and $u_{2}, v_{2} \in \widetilde{\mathcal{S}_{\varepsilon}^{0}}$ such that $u=u_{1}+u_{2}, v=v_{1}+v_{2}$ and

$$
\begin{gathered}
\left\|u_{1}\right\|_{\widetilde{\mathcal{S}_{\varepsilon}^{-1}}}+\frac{1}{\beta}\left\|u_{2}\right\|_{\widetilde{\mathcal{S}_{\varepsilon}^{0}}} \leq 2\|u\|_{\mathcal{Z}_{\beta}}, \\
\left\|v_{1}\right\|_{\widetilde{\mathcal{S}_{\varepsilon}^{-1}}}+\frac{1}{\beta}\left\|v_{2}\right\|_{\widetilde{\mathcal{S}_{\varepsilon}^{0}}} \leq 2\|v\|_{\mathcal{Z}_{\beta}} .
\end{gathered}
$$

Thus one can decompose the left-hand side of (5.4) as

$$
\begin{aligned}
\left\|\mathcal{L} \partial_{x}(u v)\right\|_{\mathcal{Z}_{\beta}} & \lesssim\left\|\mathcal{L} \partial_{x}\left(u_{1} v_{1}\right)\right\|_{\widetilde{\mathcal{S}_{\varepsilon}^{-1}}}+\left\|\mathcal{L} \partial_{x}\left(u_{1} v_{2}+u_{2} v_{1}\right)\right\|_{\widetilde{\mathcal{S}_{\varepsilon}^{-1}}}+\left\|\mathcal{L} \partial_{x}\left(u_{2} v_{2}\right)\right\|_{\widetilde{\mathcal{S}_{\varepsilon}^{-1}}} \\
& =I+I I+I I I .
\end{aligned}
$$

From the estimates (3.3) and (4.1) we get

$$
I \lesssim\left\|\partial_{x}\left(u_{1} v_{1}\right)\right\|_{\mathcal{N}_{\varepsilon}^{-1}} \lesssim\left\|u_{1}\right\|_{S_{\varepsilon}^{-1}}\left\|v_{1}\right\|_{\mathcal{S}_{\varepsilon}^{-1}} \lesssim\|u\|_{\mathcal{Z}_{\beta}}\|v\|_{\mathcal{Z}_{\beta}}
$$

On the other hand, we obtain from (5.1) that

$$
I I I \lesssim T^{\nu}\left\|u_{2}\right\|_{\mathcal{S}_{\varepsilon}^{0}}\left\|v_{2}\right\|_{\mathcal{S}_{\varepsilon}^{0}} \lesssim \beta^{2} T^{\nu}\|u\|_{\mathcal{Z}_{\beta}}\|v\|_{\mathcal{Z}_{\beta}}
$$

and

$$
\begin{aligned}
I I & \lesssim T^{\nu}\left(\left\|u_{1}\right\|_{\mathcal{S}_{\varepsilon}^{-1}}\left\|v_{2}\right\|_{\mathcal{S}_{\varepsilon}^{0}}+\left\|u_{2}\right\|_{\mathcal{S}_{\varepsilon}^{0}}\left\|v_{1}\right\|_{\mathcal{S}_{\varepsilon}^{-1}}\right) \\
& \lesssim \beta T^{\nu}\|u\|_{\mathcal{Z}_{\beta}}\|v\|_{\mathcal{Z}_{\beta}} .
\end{aligned}
$$

We thus get

$$
\left\|\mathcal{L} \partial_{x}(u v)\right\|_{\mathcal{Z}_{\beta}} \lesssim\left(1+\left(\beta+\beta^{2}\right) T^{\nu}\right)\|u\|_{\mathcal{Z}_{\beta}}\|v\|_{\mathcal{Z}_{\beta}} .
$$

This ensures that (5.4) holds for $T \sim \beta^{-2 / \nu} \leq 1$.

\section{ACKNOWLEDGEMENT}

The first author was partially supported by the ANR project "Equa-Disp". 


\section{REFERENCES}

[1] D.Bekiranov, The initial-value problem for the generalized Burgers' equation, Diff. Int. Eq. 9 (6) (1996), pp. 1253-1265. MR.1409926 (97k:35219)

[2] I. Bejenaru and T. Tao, Sharp well-posedness and ill-posedness results for a quadratic non-linear Schrödinger equation, J. Funct. Anal. 233 (2006), no. 1, 228-259. MR2204680 (2007i:35216)

[3] J. Bourgain, Fourier transform restriction phenomena for certain lattice subsets and application to nonlinear evolution equations II. The KdV equation, GAFA 3 (1993), pp. 209-262. MR:1215780 (95d:35160b)

[4] J. Bourgain, On the Cauchy problem for the Kadomtsev-Petviashvili equation, GAFA 3 (1993), 315-341. MR1223434 (94d:35142)

[5] J. Bourgain, Periodic Korteveg de Vries equation with measures as initial data, Sel. Math. New. Ser. 3 (1993), pp. 115-159. MR1466164 (2000i:35173)

[6] M. Christ, J. Colliander and T. Tao, Asymptotics, frequency modulation, and low regularity ill-posedness for canonical defocusing equations, Amer. J. Math. 125 (2003), no. 6, 1235-1293. MR2018661 (2005d:35223)

[7] J. Colliander, M. Keel, G. Staffilani, H. Takaoka and T. Tao, Sharp global well-posedness results for periodic and non-periodic $K d V$ and modified $K d V$ on $\mathbb{R}$ and $\mathbb{T}$, J. Amer. Math. Soc. 16 (2003), pp. 705-749. MR.1969209 (2004c:35352)

[8] W. Chen, C. Miao and J. Li, On the well-posedness of the Cauchy problem for dissipative modified Korteweg-de Vries equations, Diff. Int. Eq. 20 (2007), no. 11, 1285-1301. MR2372427 (2008k:35401)

[9] D.B. Dix, Nonuniqueness and uniqueness in the initial-value problem for Burger's equation, SIAM J. Math. Anal. 27 (3) (1996), pp. 708-724. MR.1382829 (97c:35174)

[10] P. Gérard, Nonlinear Schrödinger equations in inhomogeneous media: Wellposedness and illposedness of the Cauchy problem, International Congress of Mathematicians. Vol. III, 157182, Eur. Math. Soc., Zürich, 2006. MR2275675 (2007k:35459)

[11] J. Ginibre, Le problème de Cauchy pour des EDP semi-linéaires périodiques en variables d'espace (d'après Bourgain), in Séminaire Bourbaki 796, Astérique 237, 1995, 163-187. MR.1423623 (98e:35154)

[12] J. Ginibre, Y. Tsutsumi and G. Velo, On the Cauchy problem for the Zakharov system, J. Funct. Analysis, 151 (1997), no. 2, 384-436. MR.1491547(2000c:35220)

[13] T. Kappeler and P. Topalov, Global wellposedness of $K d V$ in $H^{-1}(\mathbb{T}, \mathbb{R})$, Duke Math. J. 135 (2006), no. 2, 327-360. MR2267286 (2007i:35199)

[14] C. E. Kenig, G. Ponce, and L. Vega, A bilinear estimate with applications to the KdV equation, J. Amer. Math. Soc. 9 (1996), pp. 573-603. MR.1329387 (96k:35159)

[15] L. Molinet and S. Vento, Sharp ill-posedness and well-posedness results for the KdV-Burgers equation: The real line case (to appear in Annali della Scuola Normale - Classe di Scienze).

[16] L. Molinet and F. Ribaud, On the low regularity of the Korteweg-de Vries-Burgers equation, I.M.R.N. 37 (2002), pp. 1979-2005. MR1918236 (2003e:35272)

[17] E. Ott and N. Sudan, Damping of solitary waves, Phys. Fluids 13 (6) (1970), pp. 1432-1434.

[18] T. Tao, Multilinear weighted convolution of $L^{2}$-functions, and applications to nonlinear dispersive equations. Amer. J. Math. 123 (2001), no. 5, 839-908. MR1854113 (2002k:35283)

[19] T. Tao, Scattering for the quartic generalised Korteweg-de Vries equation, J. Diff. Eq. 232 (2007), no. 2, 623-651. MR2286393 (2008i:35178)

[20] D. Tataru, On global existence and scattering for the wave maps equation, Amer. J. Math. 123 (1) (2001), 37-77. MR 1827277 (2002c:58045)

[21] S. Vento, Global well-posedness for dissipative Korteweg-de Vries equations, Funkcial. Ekvac. 54 (2011), no. 1, 119-138. MR.2829551

Laboratoire de Mathématiques et Physique Théorique, Université François Rabelais Tours, Fédération Denis Poisson-CNRs, Parc Grandmont, 37200 Tours, France

E-mail address: Luc.Molinet@lmpt.univ-tours.fr

L.A.G.A., Institut Galilée, Université Paris 13, 93430 Villetaneuse, France

E-mail address: vento@math.univ-paris13.fr 